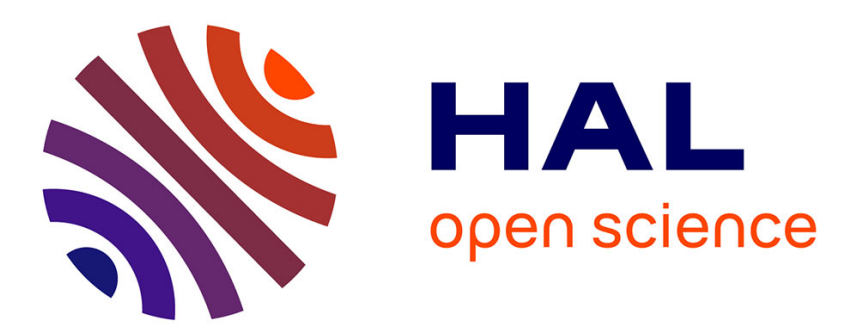

\title{
Airship gust response probabilistic model construction
}

Fabrice Poirion, Guy Mortchelewicz

\section{To cite this version:}

Fabrice Poirion, Guy Mortchelewicz. Airship gust response probabilistic model construction. EURODYN 2020, Nov 2020, ONLINE, Greece. hal-03027518

\section{HAL Id: hal-03027518 https://hal.science/hal-03027518}

Submitted on 4 Dec 2020

HAL is a multi-disciplinary open access archive for the deposit and dissemination of scientific research documents, whether they are published or not. The documents may come from teaching and research institutions in France or abroad, or from public or private research centers.
L'archive ouverte pluridisciplinaire HAL, est destinée au dépôt et à la diffusion de documents scientifiques de niveau recherche, publiés ou non, émanant des établissements d'enseignement et de recherche français ou étrangers, des laboratoires publics ou privés. 


\title{
AIRSHIP GUST RESPONSE PROBABILISTIC MODEL CONSTRUCTION
}

\author{
F. Poirion ${ }^{1}$ and G. Mortchelewicz ${ }^{1}$ \\ ${ }^{1}$ ONERA, Université Paris Saclay \\ poirion@onera.fr, guy.mortchelewicz@onera.fr
}

Keywords: Airship, Gust, Probabilistic surrogate, Karhunen Loeve.

\begin{abstract}
In the context of random environment, time-domain simulation of structural responses is often necessary when the structure is nonlinear, but not only: when the structure is linear and the excitation modelled as a non-stationnary non-Gaussian process, Monte Carlo approaches are necessary to identify the probabilistic distribution of the output. In this paper we use a stochastic model developped for non-stationnary and non-Gaussian random processes. It is based on an empirical Karhunen-Loeve expansion of the process constructed from an available data basis. We study the particular case of linear structures: the Monte Carlo becomes particularely simple: one needs only to consider the construction of a low number of responses, corresponding to the deterministic eigen functions of the Karhunen-Loeve expansion, hence avoiding high number of calls to industrial codes. The KL expansion of the response is then obtained directly using these responses and the system output probabilistic characterization can be constructed through Monte Carlo simulation of the KL output model. This approach is used in order to quantify the effect of gusts on a future airship using a database obtained during a winter storm from which a model of vertical gusts isconstructed. Response comparisons for realistic and gusts and the one prescribed by national regulation requirements will be shown.
\end{abstract}




\section{INTRODUCTION}

There exists today a new interest in airship exploitation as appears a need for a safe and sustainable solution that can deliver heavy cargo or personnel to remote communities that have no infrastructures. For instance the Flying Whales start-up has launched a 60 ton capacity airship project which size is unusual: $150 \mathrm{~m}$ long, $60 \mathrm{~m}$ large and $40 \mathrm{~m}$ height.

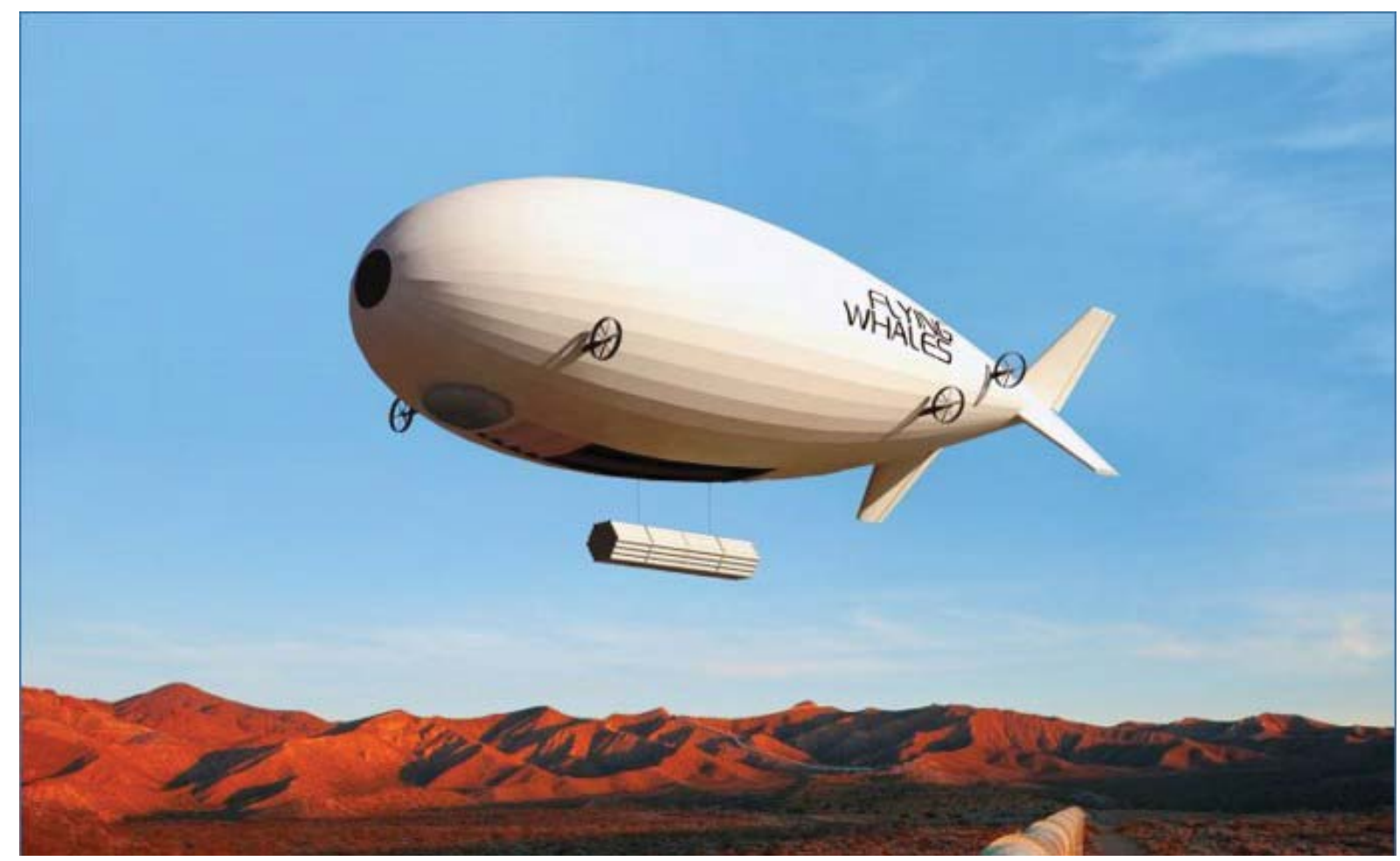

Figure 1: Flying Whale LCA60T airship project. Source: Flying Whales

New regulations had to be specified in order to certify such modern airships and among them was introduced one dedicated to gust. In the TAR (Transport Airship Regulation) the gust is modeled as an harmonic excitation and the question which arises is how will the airship response to real gusts, which exist within a turbulent wind field, compare to the regulation gust response. Real gusts are random phenomena which can be modeled as non-stationary and non-Gaussian processes. Therefore one needs to characterize the output of the airship to this random excitation. Airship is modeled as a coupled aerodynamic/structure system and due to the very low Mach number involved in airship flight the use of a linearized aerodynamic code is entirely justified. Nevertheless the response characterization goes through a complete Monte-Carlo approach since the input is non-stationary and non-Gaussian which can render the methodology numerically very expensive for high dimension structural models. We show that when the input process can be represented by its Karhunen Loeve expansion, the linearity of the numerical code allows to construct directly the Karhunen Loeve expansion of the response which can be used to generate samples used for constructing various estimators of the response. An illustration will be given on a simplified airship model which will be subject to real gusts identified in given wind measures. 


\section{PROBABILISTIC MODEL CONSTRUCTION OF OBSERVED SAMPLE PATHS}

Three dimensional wind data are relatively scarce and existing gust databases contain insufficient samples in order to be relevant in a Monte Carlo procedure. An enrichment method will therefore be used in order to generate supplementary synthetic samples. This method, based on Karhunen Loeve expansion of stochastic process, is described in [10, 7]. We shall briefly recall the main ingredients.

Karhunen-Loève (KL) expansion provides a suitable framework for modeling a non-stationary scalar random process $X(t, \omega)$ :

$$
\forall t \in \mathcal{D}, X(t)=\lim _{N \rightarrow+\infty} \sum_{\alpha=1}^{N} \sqrt{\lambda_{\alpha}} \xi_{\alpha} \phi_{\alpha}(t),
$$

in which $\xi_{1}, \xi_{2}, \ldots, \xi_{\alpha}, \ldots$ are uncorrelated random variables given by

$$
\xi_{\alpha}=\frac{1}{\sqrt{\lambda_{\alpha}}} \int_{\mathcal{D}}<X(t), \phi_{\alpha}(t)>d t
$$

where the $\lambda_{\alpha}$ and $\phi_{\alpha}(t)$ are the solutions of the integral equation

$$
\int_{\mathcal{D}} R_{X}\left(t, t^{\prime}\right) \phi\left(t^{\prime}\right) d t^{\prime}=\lambda \phi(t)
$$

and where the limit in $(1)$ is taken in the space $L^{2}(\Omega, \mathbb{R})$.

Let be given $\mathcal{N}$ measures $\left\{X^{(l)}\left(t_{i}\right) ; i=1, \ldots, N\right\} ; l=1, \mathcal{N}$ of a random phenomenon sample path. The first step to the KL expansion construction is to estimate the empirical autocorrelation of the underlying process $X$ :

$$
R\left(t_{i}, t_{j}\right)=\frac{1}{\mathcal{N}} \sum_{l=1}^{\mathcal{N}} X^{(l)}\left(t_{i}\right) X^{(l)}\left(t_{j}\right)
$$

Solving the discretized eigenvalue problem (3) yields the $\lambda_{\alpha}$ and $\phi_{\alpha}\left(t_{i}\right)$, for $\alpha, i=1, N$.

The second step is to construct the samples $\xi_{\alpha}^{(l)}$ using relation (2) which gives an explicit relation :

$$
\xi_{\alpha}^{(l)}=\frac{1}{\sqrt{\lambda_{\alpha}}} \sum_{i=1}^{N} X^{(l)}\left(t_{i}\right) \phi_{\alpha}\left(t_{i}\right) \Delta t ; l=1, \mathcal{N} ; \alpha=1, N
$$

where $\Delta t$ is the sampling time step.

In order to be able to use the KL expansion for simulating the random process $X$, the last step is to identify the distribution of the random vector $\xi=\left(\xi_{1}, \ldots, \xi_{N}\right)$, which in the general case is not Gaussian. This multivariate distribution will be approximated by a Gaussian kernel density estimator :

$$
\hat{f}_{\mathcal{N}, h}(x)=\frac{1}{\mathcal{N} h^{N}(2 \pi)^{N / 2}} \sum_{\ell=1}^{\mathcal{N}} \exp \frac{\left(x-\xi^{(\ell)}\right)^{T}\left(x-\xi^{(\ell)}\right)}{2 h^{2}}
$$

where $\xi^{(\ell)}, \ell=1, \mathcal{N}$ are the observed samples of random variable $\xi$.

Details on kernel density estimators construction can be found in the following references $[3,9,8]$.

Simulation of a multivariate random variable $\xi$ described by the kernel density (6) is straightforward: 
- generate a random integer $J$ uniformly distributed on $\{1,2, \ldots, \mathcal{N}\}$

- generate a $\mathcal{N}$-dimensional normalized Gaussian random variable $\mathcal{G}$

- construct the sample $\xi=\xi^{(J)}+h \times \mathcal{G}$.

In practice, the approximation due to truncation of the KL expansion is evaluated considering the total energy $\int_{[0, T]} E\left(X(t)^{2}\right) d t$ of the process : $\int_{[0, T]} R_{X}(t, t) d t=\sum_{\alpha} \lambda_{\alpha}$, the relative error $\epsilon$ being then defined by:

$$
\epsilon=\sum_{\alpha=1}^{N_{T}} \lambda_{\alpha} / \sum_{\alpha=1}^{\infty} \lambda_{\alpha}=\sum_{\alpha=1}^{N_{T}} \lambda_{\alpha} / \int_{[0, T]} R_{X}(t, t) d t
$$

Numerically, the total energy can be determined from the empirical autocorrelation function of the $N$ trajectories:

$$
\int_{[0, T]} R_{X}(t, t) d t=\sum_{i=1}^{N} R_{X}\left(t_{i}, t_{i}\right) \Delta t_{i}
$$

Finally one has to characterize the response of a system to a random excitation modeled as a KL expansion:

$$
X(t, \omega)=\sum_{\alpha=1}^{M} \sqrt{\lambda_{\alpha}} \xi_{\alpha}(\omega) \phi_{\alpha}(t) \rightarrow \longrightarrow
$$

When the system is linear it is possible to use the input expression linearity to construct directly a probabilistic model of the system output.

\section{RESPONSE OF A LINEAR DYNAMICAL SYSTEM TO KL EXCITATION}

There exist many real-life systems which are described using industrial linear codes: in structures, aeronautics, etc. The use of such linear approximations of real structures and systems are still today mandatory in the conception phase or for optimization because high fidelity models are too CPU intensive. This is particularly true for probabilistic analysis based on Monte Carlo approaches. Even in the case of linear systems, there exist no analytic methods to characterize the system output when the excitation is modeled through a non-Gaussian non-stationary process. One has to go through numerical simulations in order to construct various statistical estimators.

Consider a linear system described through a differential equation

$$
Y^{\prime}(t)=A(t) Y(t)+X(t) ; Y(0)=Y_{0} ; t \in[0, T] .
$$

It is well known that its solution can be written

$$
Y(t)=H(t, 0) Y_{0}+\int_{0}^{t} H(t, s) X(s) d s
$$

where $H$ is the resolvent matrix. Function $H$ is the impulse response function and represents the linear system. It is generally obtained using an industrial code (Nastran, Marc, ...) and its derivation can be computationally expensive for high dimension models such as the ones encountered in aeronautics. When $X$ is a general second order stochastic process defined on 
a given probability space $(\Omega, \mathcal{T}, P)$, Monte Carlo approaches have to be used in order to construct statistical estimators of $Y$, and, even in a linear context, Monte Carlo based probabilistic analysis can become non practical for some applications. When $X$ is approximated through a truncated KL expansion denoting $m_{X}(t)$ its expectation, relation (9) can be written,

$$
\begin{aligned}
Y(t, \omega)=H(t, 0) Y_{0}+\int_{0}^{t} H(t, s) m_{X}(s) d s+ \\
\qquad \sum_{\alpha=1}^{N} \sqrt{\lambda_{\alpha}} \xi_{\alpha} \int_{0}^{t} H(t, s) \phi_{\alpha}(s) d s, ; t \in[0, T]
\end{aligned}
$$

The output characterization necessitates only $N+2$ calls of the linear codes, whith $N$ generally less than 100 in order to construct the $N$ deterministic response functions $\psi_{\alpha}(t)=$ $\int_{0}^{t} H(t, s) \phi_{\alpha}(s) d s$, the initial condition and the mean excitation response. The probabilistic analysis is then achieved generating random linear combinations of these $N$ functions.

\section{AIRSHIP AEROELASTIC MODEL}

The illustrations given in the following involve an airship model of dimensions $150 \times 60 \times 44$ meters and a weight of 60 tons. The cruise flight altitude is 1000 meter at a speed of $100 \mathrm{~km} / \mathrm{h}$. A crude finite element model, sufficiently meaningful for the aeroelastic illustration is used and is represented on figure 2.

The coupled system airship/aerodynamic forces is modeled using the code CAPRI [4, 5] which uses a linearized aerodynamic model based on the doublet lattice method [1]. The use of a linearized model is totally justified for an airship due to the very low Mach number involved: Mach 0.086. The aerodynamic force due to a gust is given in the frequency domain by the relation:

$$
\hat{F}(\omega)=T(\omega) \times \frac{\hat{W}(M, \omega)}{V}
$$

where $V$ is the airship speed, $\hat{W}(M, \omega)$ the Fourier transform of a gust and $T(\omega)$ the transfer function calculated by the CAPRI code for a set of frequency values. Using a rational approximation of the transfer function allows to build a time domain representation of the force, solving a differential equation [6]. What is considered for certification is the charge factor defined by:

$$
N(t)=\frac{F(t)}{M g},
$$

where $M$ is the airship mass and $g$ the standard gravity.

\section{GUST MODELS}

The transportation airship requirements imply that the airship structure integrity should not be affected when the airship encounters an isolated gust defined by the following expression

$$
v_{g}=\frac{V_{g}}{2}\left(1+\cos \left(\pi \frac{x-x_{g}}{L_{g}}\right)\right) \text { for }\left|x-x_{g}\right| \leq L_{g}
$$

where $v_{g}$ is the gust speed at the point of coordinate $x, x_{g}$ is the coordinate of the gust center, $V_{g}$ is the maximal gust speed located at point $x_{g}, L_{g}$ is the gust half length.

Such a gust is of course not representative of real gusts as it will be shown later on. Therefore it is interesting to compare the real gust and the regulation gust effects on the structure. First 


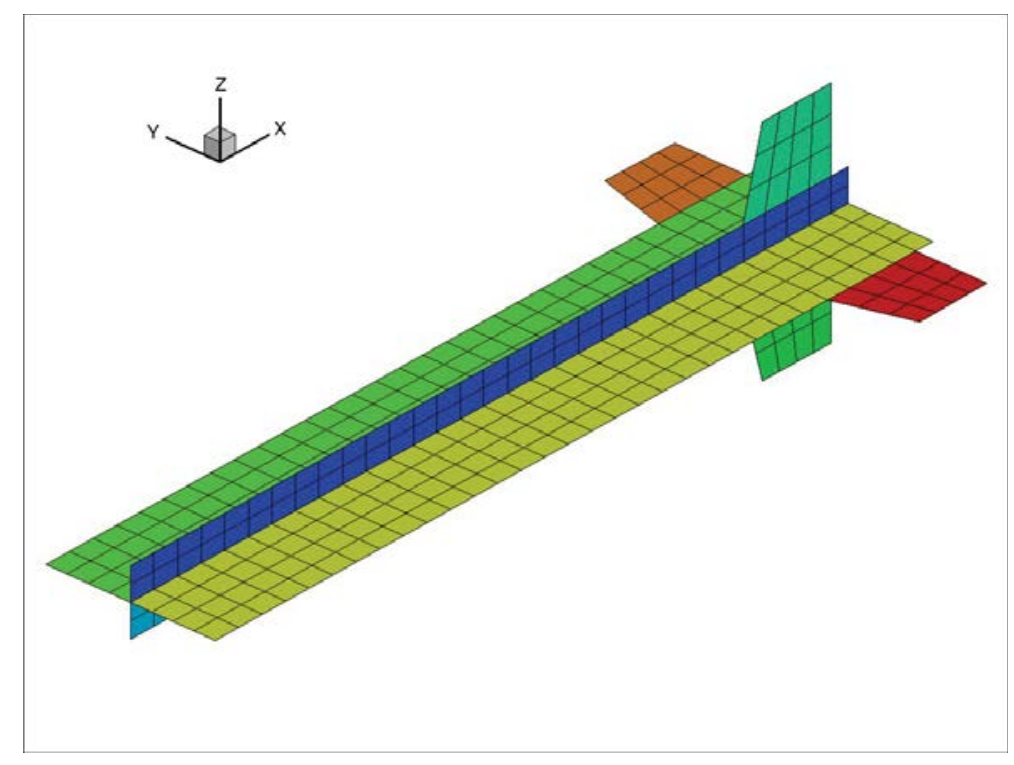

Figure 2: Airship finite element model

it is necessary to define what is a gust: a gust is a short-term wind speed variation within a turbulent wind field. There exist several methods to detect gusts in a wind time history [2], in this application we shall consider what is called the velocity increment method: a moving window of length $T$ is shifted along the wind time history $W(t)$ and a gust of amplitude $V_{g}$ is detected when $W(T)-W(0)=V_{g}$. There exist several public wind databases available, here

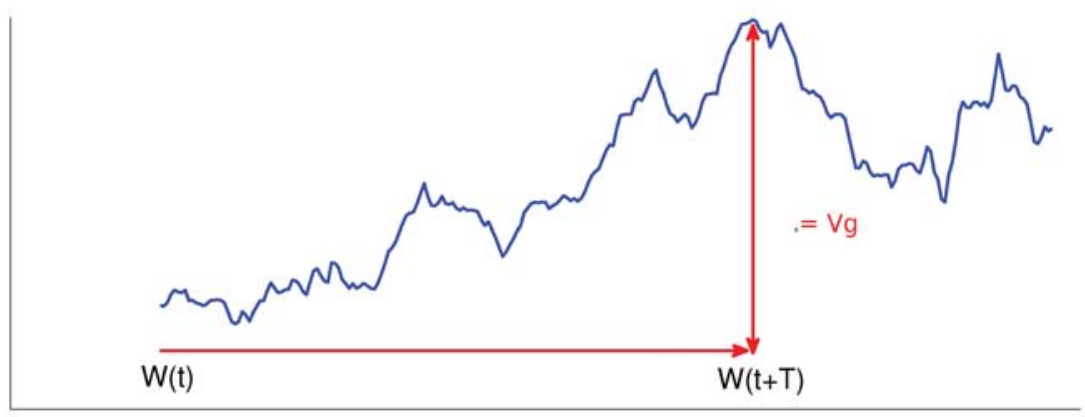

Figure 3: Velocity increment gust detection

we shall use a wind data obtained in a FINO offshore research platform ${ }^{1}$ located in the North sea during a winter storm. The three components of the wind have been recorded during three days. Figure 4 shows the longitudinal and vertical component of the wind time history. This last component will be used in order to detect gusts.

\section{NUMERICAL ILLUSTRATION}

The transportation airship requirements fix the maximum gust speed $V_{g}$ to $7.6 \mathrm{~m} / \mathrm{s}$ for an airship speed equal to $100 \mathrm{~km} / \mathrm{h}$. The gust half length $L_{g}$ is chosen equal to $75 \mathrm{~m}$ (the airship

\footnotetext{
${ }^{1}$ FINO: Research platforms in the North Sea and in the Baltic Sea, GL-Windenergie, Hamburg (http://www.fino-offshore.de)
} 

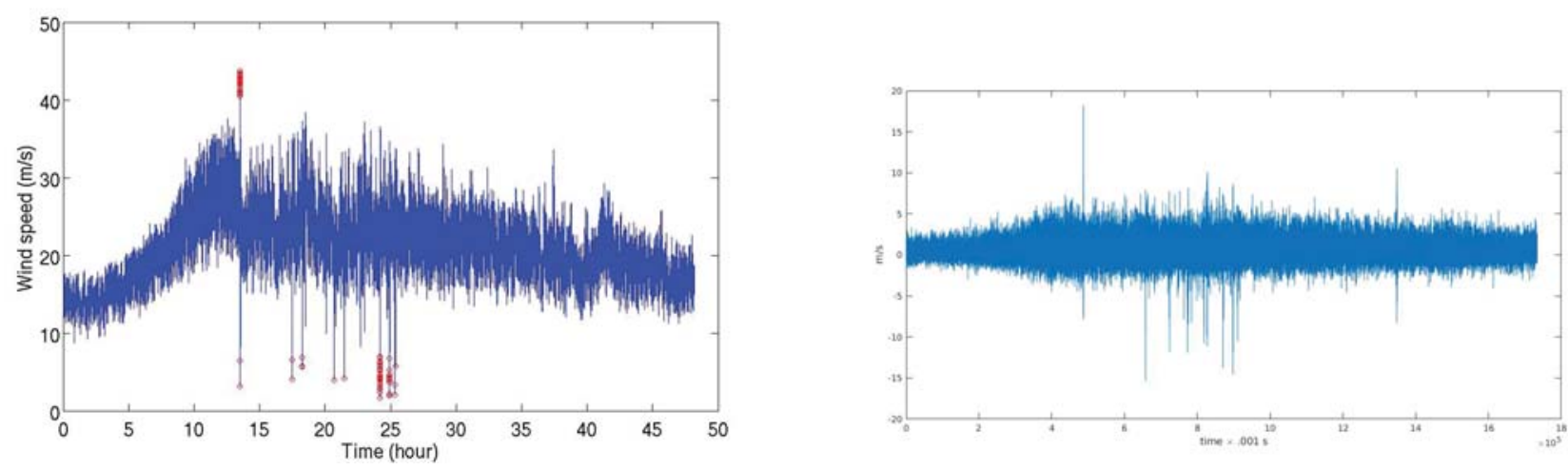

Figure 4: Horizontal and vertical wind time history

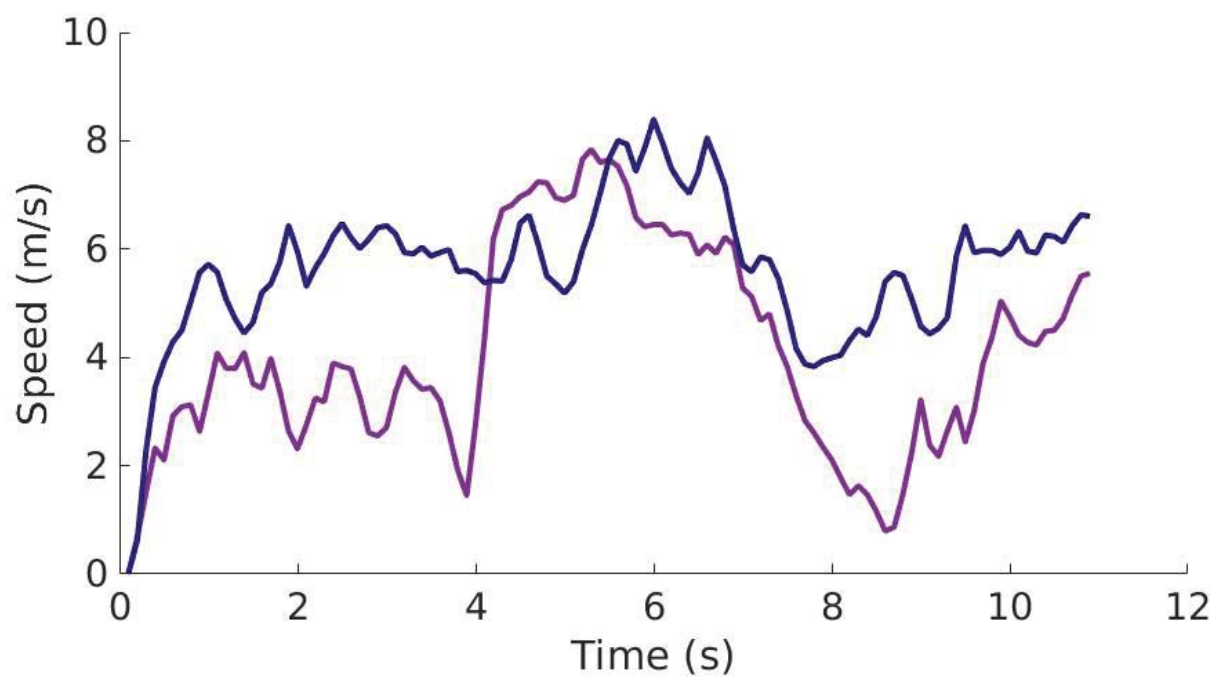

Figure 5: Two detected gusts

length being equal to $150 \mathrm{~m}$ ). In a first stage we shall detect gusts characterized by the above values of $V_{g}$ and $L_{g}$ in the recorded vertical wind time history. Thirty nine gusts are detected and figure 5 shows two of them. Figure 6 compares the mean real gust and the TAR prescribed gust.

\subsection{Input simulation}

In a second stage the Karhunen Loeve stochastic model of the vertical gusts is constructed using the thirty nine gusts in the database. Thirty four terms are kept in the expansion. Using this model, 5000 gust sample paths are generated, each leading to the calculation of the corresponding charge factor. Figure 7 shows the mean charge factor as well the $\pm \sigma$ dispersion due to the generated gusts compared to the charge factor due to the TAR requirement gust, figure 8 gives the mean number of crossings of the charge factor for different levels. 


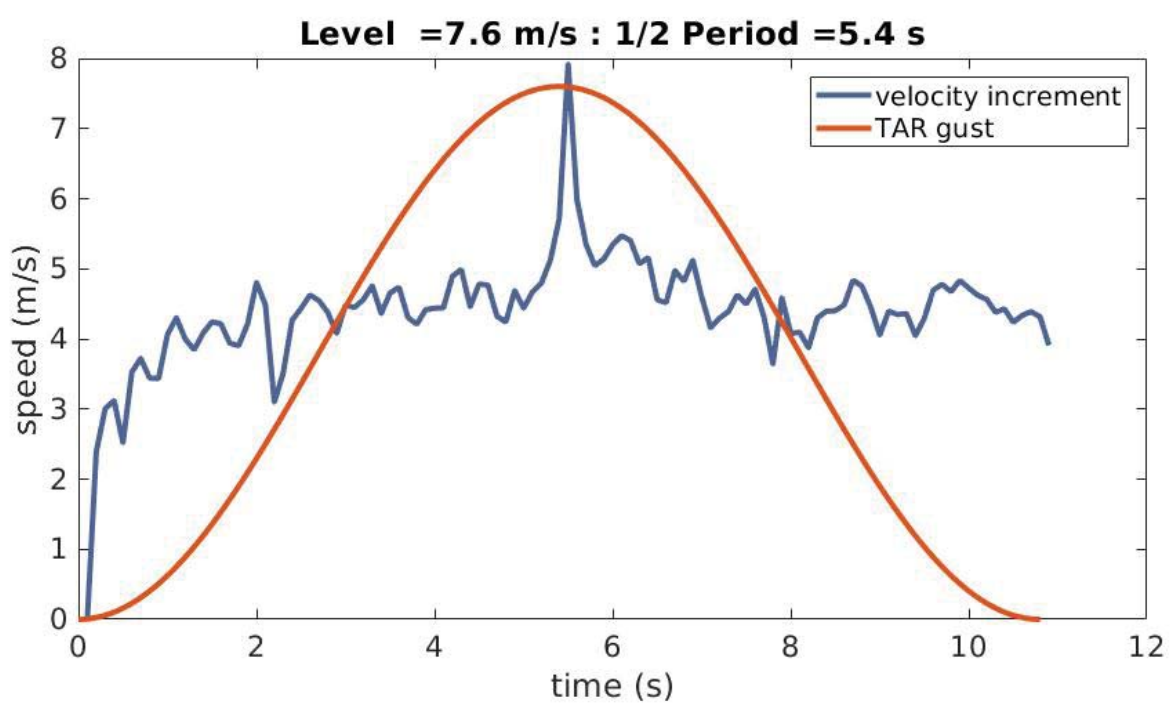

Figure 6: Mean gusts

\subsection{Output simulation}

We construct now the image of the gust Karhunen Loeve expansion by the linear aeroelastic operator: the input of the operator is the expansion eigen functions and the mean gust. The initial condition in equation 8 is 0 . Relation 10 yields the Karhunen Loeve expansion of the random charge factor which can be used to generate additional responses in order to construct various statistical estimates. Again, 5000 simulated responses of the charge factor are generated using the KL image expansion and the same estimators as above are constructed. Figure 9 shows the mean charge factor as well the $\pm \sigma$ dispersion due to the simulated charge factor and, of course, the result is the same as the one shown on figure 7. Figure 10 shows the mean number of crossings of the charge factor for different levels calculated using the input stochastic model or the output stochastic model: the results are identical.

\section{CONCLUSION}

We have shown in this paper that when a linear system is excited by a stochastic process represented by its Karhunen Loeve expansion, one could directly construct the Karhunen Loeve expansion of any system output, allowing its use for direct Monte Carlo characterization. An illustration has been given related to the identification of an airship response to wind gusts. The gust are represented by a Karhunen Loeve expansion constructed from a database containing turbulent wind time history measures. Results haves shown in particular the important output (charge factor) scattering compared to the one obtained using the transport air requirements.

\section{REFERENCES}

[1] E. Albano and W. Rodden. A doublet lattice method for calculating lift distributions on surfaces in subsonic flows. AIAA Journal, 7(2):279-285, Feb 1969. Errata vol 7, no. 11 Nov. 1969.

[2] E. Branlard. Wind energy: On the statistics of gusts and their propagation through a wind farm. ECN-Wind-Memo-09, 2009. 


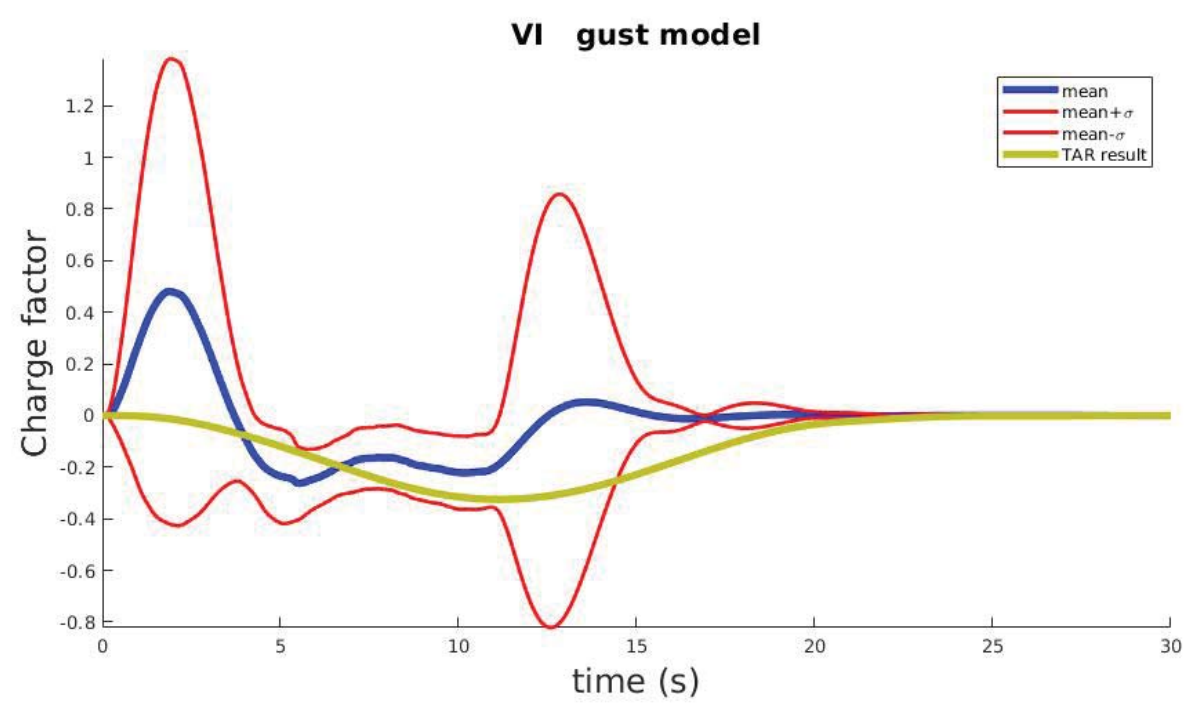

Figure 7: Mean charge factor

[3] L. Devroye and L. Györfi. Nonparametric density estimation : the $L_{1}$ view. John Wiley, New York, 1985.

[4] G. Mortchéléwicz. Prediction of aircraft transonic aeroelasticity by the linearised Euler equations. In 41th Israel annual conference Aerospace Science, february 2001.

[5] G. Mortchéléwicz. CAPRI user guide. techreport RT 1/16459, ONERA, 2009.

[6] F. Poirion. On some stochastic methods applied to aeroservoelasticity. Aerospace Science and Technology, 4:pp 201-214, 2000.

[7] F. Poirion and I. Zentner. Stochastic model construction of observed random phenomena. Probabilistic Engineering Mechanics, 36:63-71, 2014. doi 10.1016/j.probengmech.2014.03.005.

[8] D. W. Scott. Multivariate Density Estimation: Theory, Practice, and Visualization. 1992.

[9] B.W. Silverman. Density Estimation for Statistics and Data Analysis. Chapman \& Hall, 1986.

[10] I. Zentner and F. Poirion. Enrichment of seismic ground motion data basis using Karhunen-Loève expansion. Earthquake Engineering and Structural Dynamics, 41(14):1945-1957, 2012. 


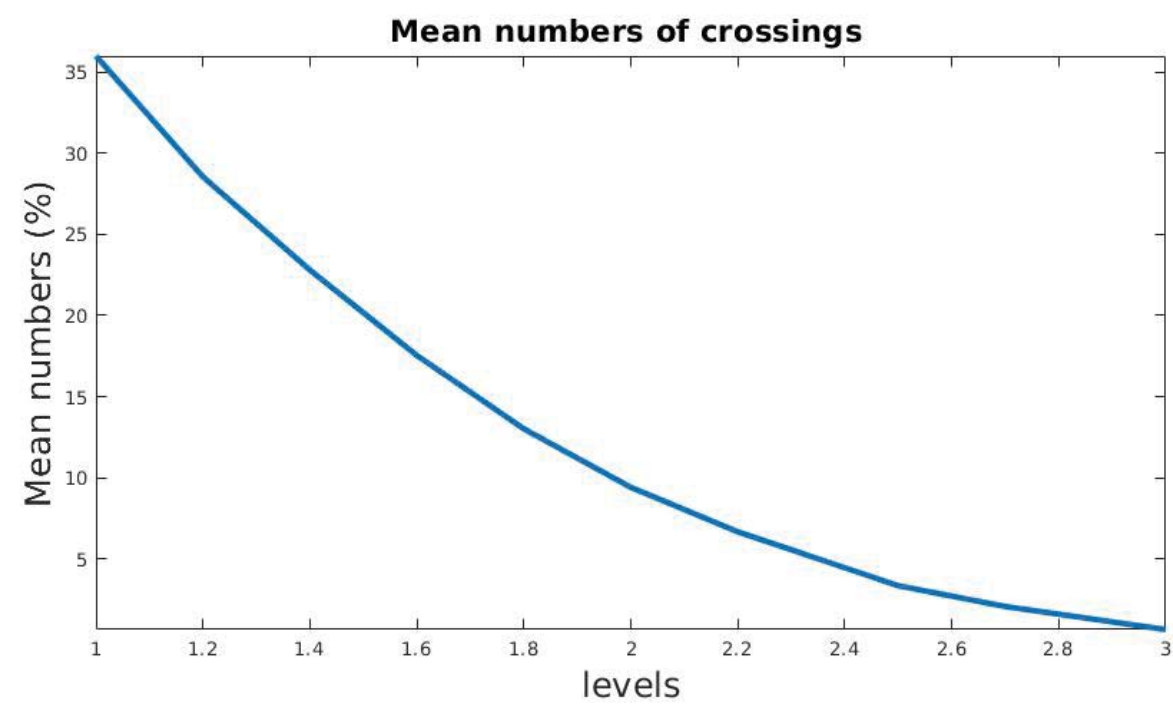

Figure 8: Mean number of crossings

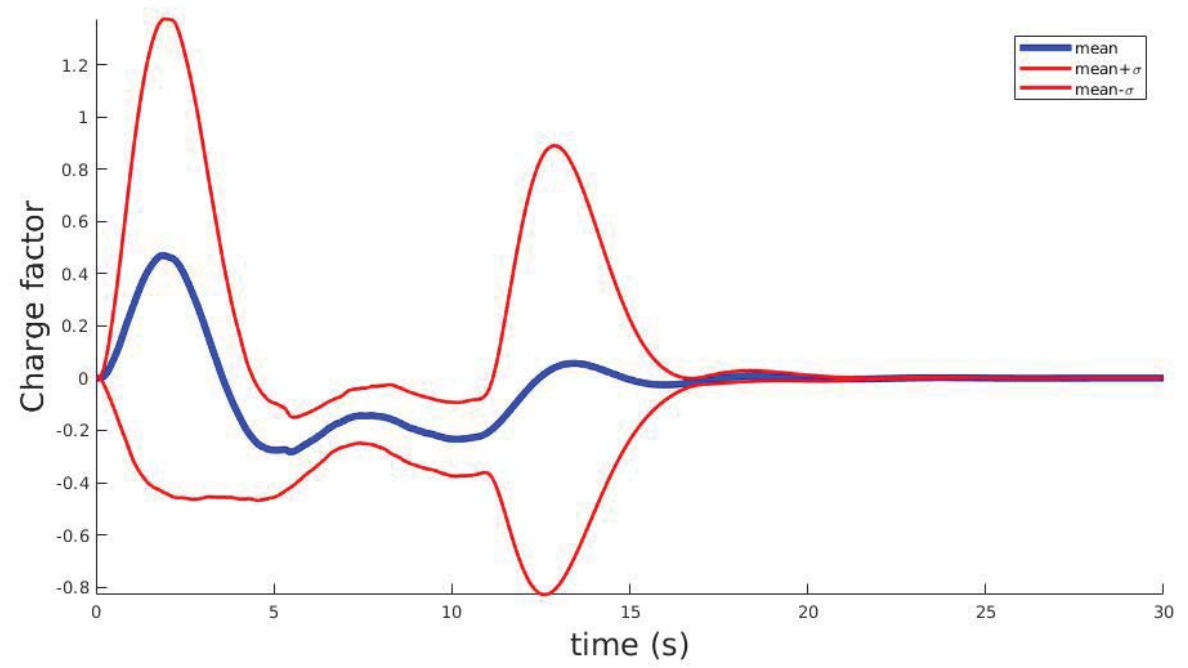

Figure 9: Mean charge factor 


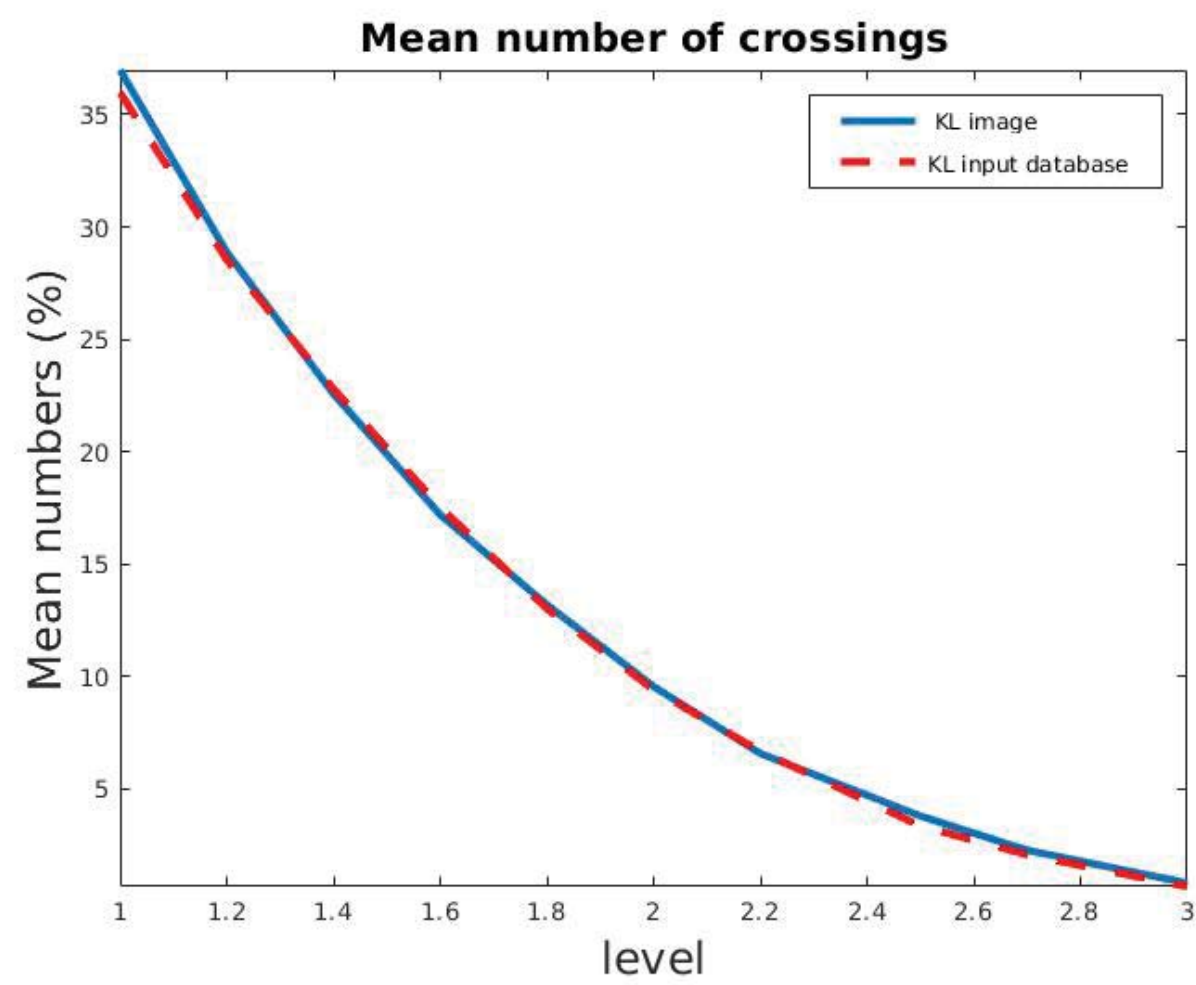

Figure 10: Mean number of crossings 